\title{
Aspectos de la biología y dinámica poblacional del pargo coliamarillo Lutjanus argentiventris en el Parque Nacional Natural Gorgona, Colombia
}

\author{
Paola A. Rojas ${ }^{1}$, Carlos F. Gutiérrez ${ }^{1}$, Vladimir Puentes ${ }^{1,2}$, \\ Angel A. Villa ${ }^{1}$ \& Efraín A. Rubio ${ }^{2}$ \\ ${ }^{1}$ Unidad Administrativa Especial del Sistema de Parques Nacionales Naturales, UAESPNN, \\ Territorial Sur-Occidente, Av. 3 G No. 37N 70, Cali, Colombia \\ ${ }^{2}$ Universidad del Valle, Facultad de Ciencias, Departamento de Biología, \\ Apartado Aéreo 25360, Cali, Colombia
}

\begin{abstract}
RESUMEN. El pargo coliamarillo Lutjanus argentiventris (Peters, 1869) fue capturado durante faenas experimentales de pesca realizadas en el Parque Nacional Natural Gorgona (Colombia) entre marzo de 1997 y diciembre de 2000. La talla promedio de captura (longitud total, LT) fue de $482 \mathrm{~mm}$ y la proporción machos: hembras fue de 1:1,2. Se observaron hembras en avanzado estado de madurez sexual durante todo el tiempo y no se observó un patrón claro de máximos reproductivos. La talla promedio de madurez sexual fue de $515 \mathrm{~mm} \mathrm{LT}$. El análisis de la relación longitudpeso no mostró diferencias significativas entre sexos y estuvo dada por la ecuación $P T=\left(6,259 * 10^{-6}\right) L T^{3,12}$. Se estimaron los parámetros de crecimiento para machos y hembras, en el período de El Niño y el período de La Niña. Las ecuaciones de crecimiento de Von Bertalanffy estimadas para la especie, en general, fueron $L T=950\left(1-e^{(-0,20(t+0,72))}\right)$ y $P T=12218\left(1-e^{(-0,20(t+0,72))}\right)^{3,12}$. La mortalidad natural estimada de acuerdo con Ralston (1987) y Pauly (1980) fue 0,43 y 0,45 respectivamente. La CPUE muestra máximos de abundancia en mayo y septiembre, y una disminución drástica de la abundancia el año 2000. En general hay claras diferencias entre las poblaciones de L. argentiventris de México y Gorgona, Colombia.
\end{abstract}

Palabras clave: Lutjanus argentiventris, biología, dinámica poblacional, El Niño, La Niña, Gorgona-Colombia.

\section{Aspects of the biology and population dynamics of the yellowtail snapper Lutjanus argentiventris in the Gorgona National Natural Park, Colombia}

\begin{abstract}
Yellowtail snapper Lutjanus argentiventris (Peters, 1869) was captured during experimental fishing trips around the Gorgona National Natural Park, Colombia, from March 1997 to December 2000. Average capture size (total length, TL) was $482 \mathrm{~mm}$ and sex ratio was 1:1.2. Advanced sexual maturity stages in females were observed during the whole study with no clear reproductive pattern; average size at sexual maturity was $515 \mathrm{~mm}$ TL. The length-weight relationship was not significantly different by sex and was described by the equation: $P T=\left(6.259 * 10^{-6}\right) . L T^{3.12}$. Growth parameters were estimated for both males and females in El Niño and La Niña periods. The Von Bertalanffy growth equations for the species in general were $L T=950\left(1-e^{(-0.20(t+0.72))}\right)$ and $P T=12218\left(1-e^{(-0.20(t+0,72))}\right)^{3.12}$. Natural mortality (M) estimations were 0.43 and 0.45, based on Ralston (1987) and Pauly (1980), respectively. CPUE showed abundance peaks in May and September, and decreased significantly in 2000. There were clear differences in parameters between the populations of L. argentiventris from Mexico and from Gorgona, Colombia.
\end{abstract}

Key words: Lutjanus argentiventris, biology, population dynamics, El Niño, La Niña, Gorgona-Colombia.

Autor corresponsal: Vladimir Puentes (vladi@wildmail.com) 


\section{INTRODUCCIÓN}

El pargo coliamarillo Lutjanus argentiventris (Peters, 1869) es un pez importante en la pesquería artesanal costera y de media altura en el océano Pacífico colombiano dado su alto valor comercial. Se distribuye en el Pacífico oriental entre el golfo de California y norte de Perú (Allen, 1985; Rubio, 1988; Franke \& Acero, 1992), en arrecifes coralinos, zonas rocosas, estuarios y desembocaduras de ríos (Rubio, 1988; Fischer et al., 1995). Es normalmente capturado con redes de arrastre, varios tipos de redes artesanales, línea de mano y línea de anzuelo de fondo (espinel) principalmente.

Entre los estudios sobre L. argentiventris se encuentra lo reportado por Fisher et al. (1995) sobre aspectos generales y taxonómicos. Álvarez (1980, 1984) reporta un espécimen sin aletas pélvicas por posibles causas congénitas Cruz-Romero et al. (1996) estudiaron varios aspectos de la biología, dinámica poblacional y pesquerías del complejo Lutjanus spp. (L. peru, L. guttatus y $L$. argentiventris) en Manzanillo y Boca de Apiza (Estado de Colima, México), y Serrano-Pinto \&
Caraveo-Patiño (1999) hicieron ensayos de cultivo de esta especie a diferentes salinidades. En Colombia cuenta con estudios realizados por Rubio (1987, 1988) y Franke \& Acero (1992) que incluyen aspectos generales y descriptivos de la especie.

El presente estudio aporta nueva información sobre la biología y dinámica poblacional de $L$. argentiventris en la zona del Parque Nacional Natural (PNN) Gorgona-Colombia, sur del Pacífico colombiano y establece diferencias (y sus posibles causas) entre las poblaciones de $L$. argentiventris de México y Gorgona.

\section{MATERIALES Y MÉTODOS}

Entre marzo de 1997 y diciembre de 2000, se realizaron faenas mensuales de pesca experimental autorizadas según el artículo 30, decreto 622 de 1977 del código de recursos naturales renovables de Colombia, las cuales se realizaron dentro y fuera de la zona marina del PNN Gorgona (Fig. 1), con espinel de 1000 anzuelos $\mathrm{N}^{\circ} 7$ y 8 , entre 40,2 y 106,4 $\mathrm{m}$ de profundidad (22 y 58 brazas, respectivamente). Se

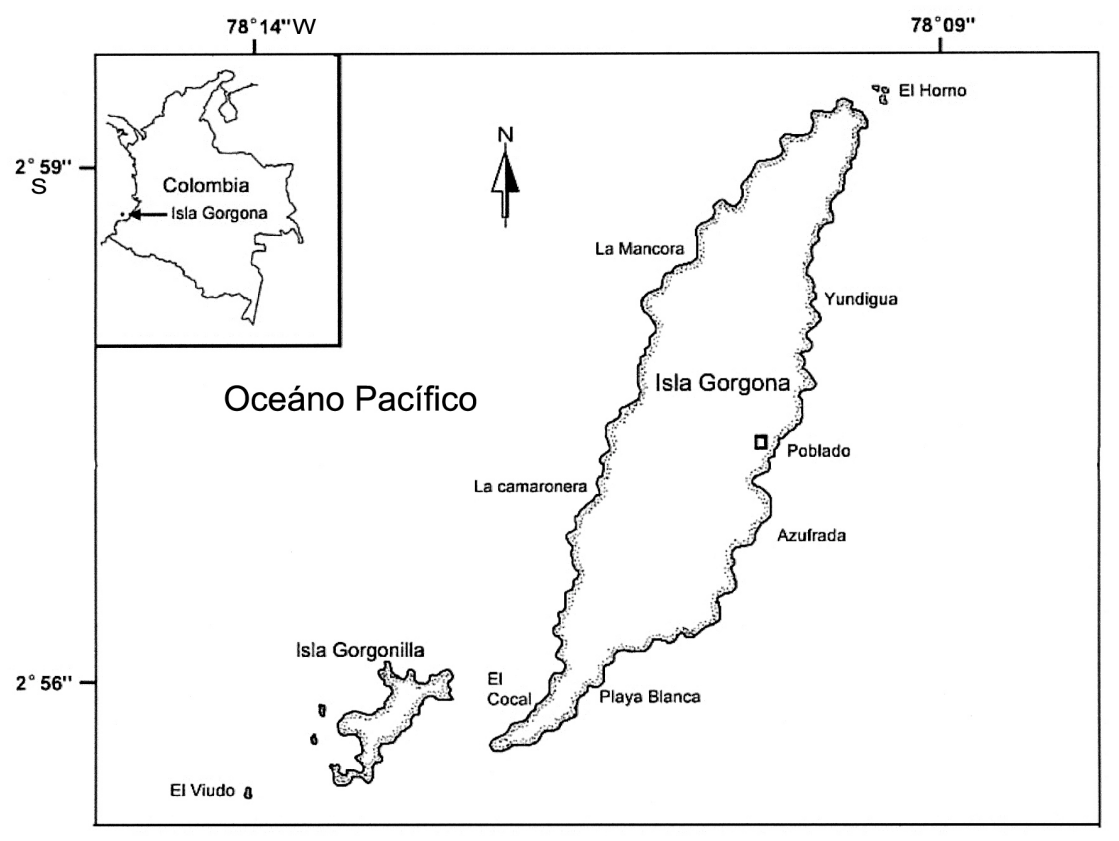

Figura 1. Parque Nacional Natural Gorgona ubicado aproximadamente a $56 \mathrm{~km}$ de la costa en la parte sur del océano Pacífico colombiano con un área de 61.687 hectáreas, de las cuales solo 1.600 hectáreas corresponden al área terrestre.

Figure 1. National Natural Park Gorgona, Colombia situated approximately $56 \mathbf{~ k m}$ from the coastline in the southern part of the Colombian Pacific Ocean, with an area of 61.687 .5 ha of which only 1.600 ha are the terrestrial portion. 
registraron datos de longitud total (LT) y longitud estándar (LS) en mm, peso total (PT), peso eviscerado (Pev), peso de gónadas (PG) y peso de hígado $(\mathrm{PH})$ en g para cada individuo. Se estimaron ecuaciones preliminares de conversión de LT a LS y viceversa, y de LT a PT y viceversa.

Se estimó la relación longitud-peso modelada por la ecuación:

$$
P T=a^{*} L T^{b}
$$

donde PT es el peso total en $\mathrm{g}$, a es una constante, LT es la longitud total en $\mathrm{mm}$, y b el exponente de la ecuación que al linealizarla se convierte en la pendiente de la recta y denota la isometría o alometría en el crecimiento.

Mediante las distribuciones mensuales de frecuencias de tallas se estimaron los parámetros de crecimiento $\mathrm{L}_{\infty}$ y $\mathrm{K}$ de la ecuación de crecimiento de Von Bertalanffy (ECVB), de machos y hembras, en el período de El Niño 1997-1998, La Niña 19981999 y el período total de estudio, utilizando el módulo ELEFAN I del Programa FAO-ICLARM Stock Assessment Tools (FISAT) (Gayanilo et al., 1996). El valor de $t_{0}$ se estimó utilizando la ecuación de Pauly (1983) para los grupos mencionados:

$$
\begin{gathered}
\log \left(-t_{0}\right)=-0,322-0,2752 * \\
\log L-1,038 * \log K
\end{gathered}
$$

donde $\mathrm{L}_{\text {es }} \mathrm{L}_{\infty}$ y $\mathrm{K}$ es la constante de crecimiento de la ECVB. $W_{\infty}$ se estimó para la especie en general, con base en las estimaciones de la ecuación (1) y las estimaciones de la ECVB para este grupo. Estimaciones de la mortalidad natural (M) se hicieron siguiendo la ecuación de regresión de Ralston (1987) para pargos y chernas:

$$
M=0,0189+2,06 * K
$$

donde $\mathrm{K}$ es la constante de crecimiento de la ECVB para el período total de estudio. M se estimó también mediante la ecuación de Pauly (1980):

$$
\begin{gathered}
\log (M)=-0,0066-0,279 * \log L+ \\
0,6543 * \log K+0,4634 * \log T
\end{gathered}
$$

donde $\mathrm{L}_{\infty}$ es la longitud asintótica de la ECVB, K es la constante de crecimiento de la ECVB y T es la temperatura promedio $\left({ }^{\circ} \mathrm{C}\right)$ para el período total de estudio dada en $27,4^{\circ} \mathrm{C}$ (Registros del Instituto de
Hidrología, Meteorología y Estudios Ambientales de Colombia, IDEAM, 2002).

El índice gonadosomático (IGS) se estimó de acuerdo a la fórmula:

$$
I G S=100 * P G / P e v
$$

donde PG es el peso de la gónada en g y Pev es el peso eviscerado en g de cada individuo. La talla promedio de madurez sexual ( $\mathrm{LT}_{\mathrm{ms}}$ ) definida como la talla promedio a la que el $50 \%$ de los peces está sexualmente maduro, se estimó siguiendo el método descrito por King (1995), ajustando una curva logística a la proporción de individuos sexualmente maduros por talla, dada por la ecuación:

$$
P=1 /(1+\exp [-r(L T-L T m s)])
$$

donde $P$ es la proporción de individuos sexualmente maduros y $r$ es la pendiente de la curva.

Complementariamente, se hicieron estimaciones y se analizó la captura por unidad de esfuerzo (CPUE) en términos de g/anzuelo, considerándolo como índice de abundancia.

\section{RESULTADOS}

L. argentiventris tiene una distribución espacial dentro y fuera de la zona marina del PNN Gorgona, especialmente en las zonas norte y sur de la isla. Un total de 603 individuos se capturaron durante el período de estudio (Tabla 1), de los cuales el 47,4\% (285) fueron hembras, 38,5\% (232) machos, 7,3\% (44) inmaduros y 6,8\% (41) que no fue posible sexar (animales mordidos o en mal estado). La proporción machos: hembras fue de 1:1,2. La talla mínima capturada fue de $200 \mathrm{~mm}$ LT (inmaduro) y la máxima de $700 \mathrm{~mm}$ LT (hembra). La talla promedio general de captura fue de $482 \mathrm{~mm}$ LT (Tabla 2).

Se observaron hembras en avanzado estado de madurez sexual durante todo el período de estudio, pero el mayor número de individuos se observó en mayo, junio, agosto, noviembre y diciembre. El IGS mostró un comportamiento errático, en donde no se evidencia una época reproducción clara a través de los años (Fig. 2). La talla media de madurez sexual varió entre los 500 y $540 \mathrm{~mm}$ LT, con un promedio de $515 \mathrm{~mm}$ en todo el período de estudio (Tabla 2).

Las ecuaciones preliminares de conversión estimadas para L. argentiventris fueron: 
Tabla 1. Número de especímenes de $L$. argentiventris analizados por mes y por año en el Parque Nacional Natural Gorgona, Colombia.

Table 1. Number of L. argentiventris specimens analyzed per month and year in the National Natural Park Gorgona, Colombia.

\begin{tabular}{|l|cccc|}
\hline & \multicolumn{4}{|c|}{ AÑ } \\
Mes & $\mathbf{1 9 9 7}$ & $\mathbf{1 9 9 8}$ & $\mathbf{1 9 9 9}$ & $\mathbf{2 0 0 0}$ \\
\hline Ene & & 7 & 1 & 0 \\
Feb & & 22 & 3 & 0 \\
Mar & 9 & $*$ & 15 & 0 \\
Abr & 4 & $*$ & 12 & 0 \\
May & 17 & 29 & 22 & 11 \\
Jun & 12 & 9 & 1 & 16 \\
Jul & 30 & 8 & 15 & 0 \\
Ago & 31 & 7 & 18 & 2 \\
Sep & 86 & 23 & 20 & 0 \\
Oct & 51 & 0 & 29 & 2 \\
Nov & 17 & 3 & 20 & 10 \\
Dic & 12 & 28 & 0 & 1 \\
\hline
\end{tabular}

* No se realizaron muestreos
Tabla 2. Talla promedio de captura, talla media de madurez sexual y CPUE por año de $L$. argentiventris en el Parque Nacional Natural Gorgona, Colombia.

Table 2. Mean capture length, mean length at sexual maturity, and CPUE by year of $L$. argentiventris at the National Natural Park Gorgona, Colombia.

\begin{tabular}{|cccc|}
\hline Año & $\begin{array}{c}\text { Talla media } \\
\text { de captura } \\
(\mathbf{m m}, \mathbf{L T})\end{array}$ & $\begin{array}{c}\text { Talla media de } \\
\text { madurez sexual } \\
(\mathbf{m m}, \mathbf{L T})\end{array}$ & $\begin{array}{c}\text { CPUE } \\
(\mathrm{g} / \text { anzuelo })\end{array}$ \\
\hline 1997 & 480 & 540 & 48,85 \\
1998 & 488 & 520 & 20,17 \\
1999 & 482 & 500 & 23,76 \\
2000 & 477 & 500 & 4,53 \\
\hline Promedio & 482 & 515 & 24,33 \\
\hline
\end{tabular}

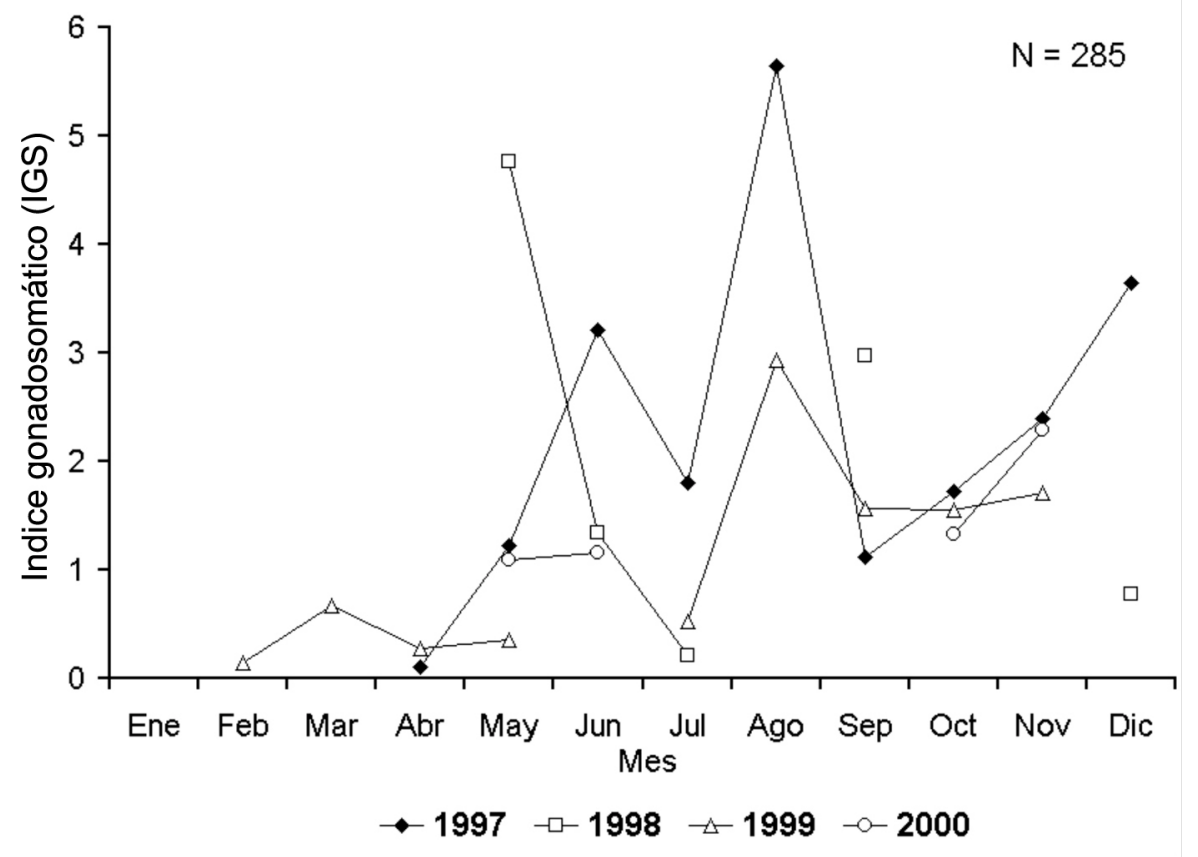

Figura 2. Indice gonadosomático de L. argentiventris en el Parque Nacional Natural Gorgona, Colombia. N: número de individuos.

Figure 2. Gonadosomatic index of L. argentiventris in the National Natural Park Gorgona, Colombia. N: number of individuals. 


$$
\begin{gathered}
L T=1,1229 * L S+3,3316 \\
\left(\mathrm{r}^{2}=0,966, \mathrm{~N}=302\right) \\
L S=0,8607 * L T-1,5576 \\
\left(\mathrm{r}^{2}=0,966, \mathrm{~N}=302\right) \\
P T=90,1527 * L T-2754,01 \\
\left(\mathrm{r}^{2}=0,887, \mathrm{~N}=562\right) \\
L T=0,0098 * P T+32,5422 \\
\left(\mathrm{r}^{2}=0,887, \mathrm{~N}=562\right)
\end{gathered}
$$

Machos y hembras se encontraron representados en casi todas las clases de tallas, por lo que no se observaron diferencias significativas entre sexos, aunque sí hubo diferencias significativas en la distribución de frecuencias de talla entre sexos (MannWhitney U Test, p < 0,001). Se observó una predominancia de machos en tallas menores (300$400 \mathrm{~mm}$ LT) y de hembras en tallas mayores (550600 mm LT) (Fig. 3).

Por otra parte, no se encontraron diferencias significativas entre las pendientes de machos y hembras en las ecuaciones linealizadas de la relación longitud-peso (ANCOVA, p > 0,05) y la relación longitud-peso para la especie, en general, estuvo dada por la ecuación:

$$
\begin{gathered}
P T=\left(6,259 * 10^{-6}\right) L T^{3,12} \\
\left(\mathrm{~N}=562, \mathrm{r}^{2}=0,936\right)
\end{gathered}
$$

La estimación de los parámetros de crecimiento en machos fueron $\mathrm{L}_{\infty}(L T)=970 \mathrm{~mm}, \mathrm{~K}=0,27 \mathrm{año}^{-1}$, $\mathrm{t}_{0}=-0,53$ años, $\mathrm{y}$ en hembras $\mathrm{L}_{\infty}(L T)=980 \mathrm{~mm}, \mathrm{~K}$ $=0,29$ año $^{-1}, \mathrm{t}_{0}=-0,49$, modelando las siguientes ecuaciones de crecimiento:

$$
\begin{gathered}
L T=970\left(1-e^{-0,27(t+0,53)}\right) \text { Machos } \\
L T=980\left(1-e^{-0,29(t+0,49)}\right) \text { Hembras }
\end{gathered}
$$

En el período de El Niño fueron $\mathrm{L}_{\infty}(L T)=970$ $\mathrm{mm}, K=0,27$ año ${ }^{-1}, \mathrm{t}_{0}=-0,53$ años. En el período de La Niña fueron $\mathrm{L}_{\infty}(L T)=950 \mathrm{~mm}, \mathrm{~K}=0,26$ $\mathrm{año} \mathrm{o}^{-1}, \mathrm{t}_{0}=-0,55$ años, las cuales modelaron las siguientes ecuaciones de crecimiento:

$$
\begin{aligned}
& L T=970\left(1-e^{-0,27(t+0,53)}\right) \text { El Niño } \\
& L T=950\left(1-e^{-0,26(t+0,55)}\right) \text { La Niña }
\end{aligned}
$$

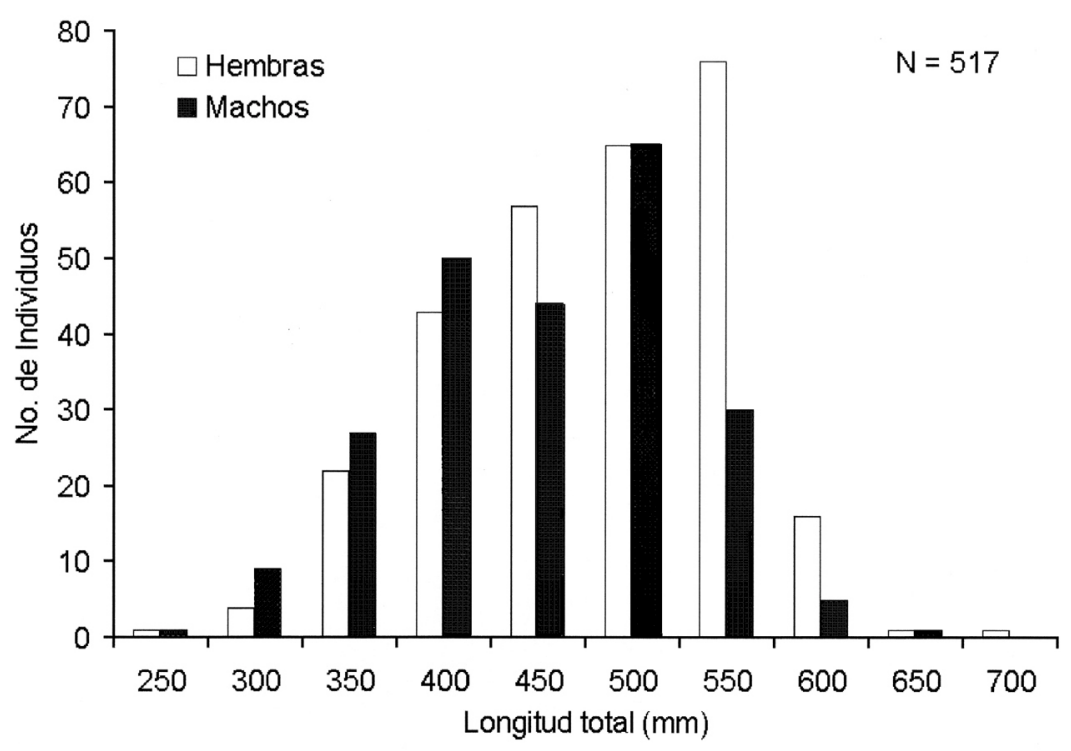

Figura 3. Distribución de frecuencias de tallas en machos y hembras de L. argentiventris en el Parque Nacional Natural Gorgona. N: número de individuos.

Figure 3. Length frequency distribution of males and females L. argentiventris in the National Natural Park Gorgona. N: number of individuals. 
Las curvas de crecimiento de machos y hembras generadas con base en las ecuaciones 12 y 13 se observan en la Figura 4a; de igual manera las curvas de crecimiento para los períodos de El Niño y La Niña, con base en las ecuaciones 14 y 15 se observan en la Figura 4b las cuales no presentan mayores diferencias entre sí. Se estimaron también los parámetros de crecimiento para la especie: $\mathrm{L}_{\infty}(L T)=950 \mathrm{~mm}, \mathrm{~K}=0,20 \mathrm{año}^{-1}, \mathrm{t}_{0}=-0,72$ años $\mathrm{y}$ $\mathrm{W}_{\infty}=12.218 \mathrm{~g}$, los cuales modelaron las siguientes ecuaciones, con sus respectivas curvas de crecimien- to ajustadas a las distribuciones mensuales de talla, generadas por el programa ELEFAN I (Fig. 5):

$$
\begin{gathered}
L T=950\left(1-e^{-0,20(t+0,72)}\right) \\
P T=12218\left(1-e^{-0,20(t+0,72))^{3,12}}\right.
\end{gathered}
$$

La mortalidad natural estimada de acuerdo con Ralston (1987) y Pauly (1980) fue de 0,43 y 0,45, respectivamente.
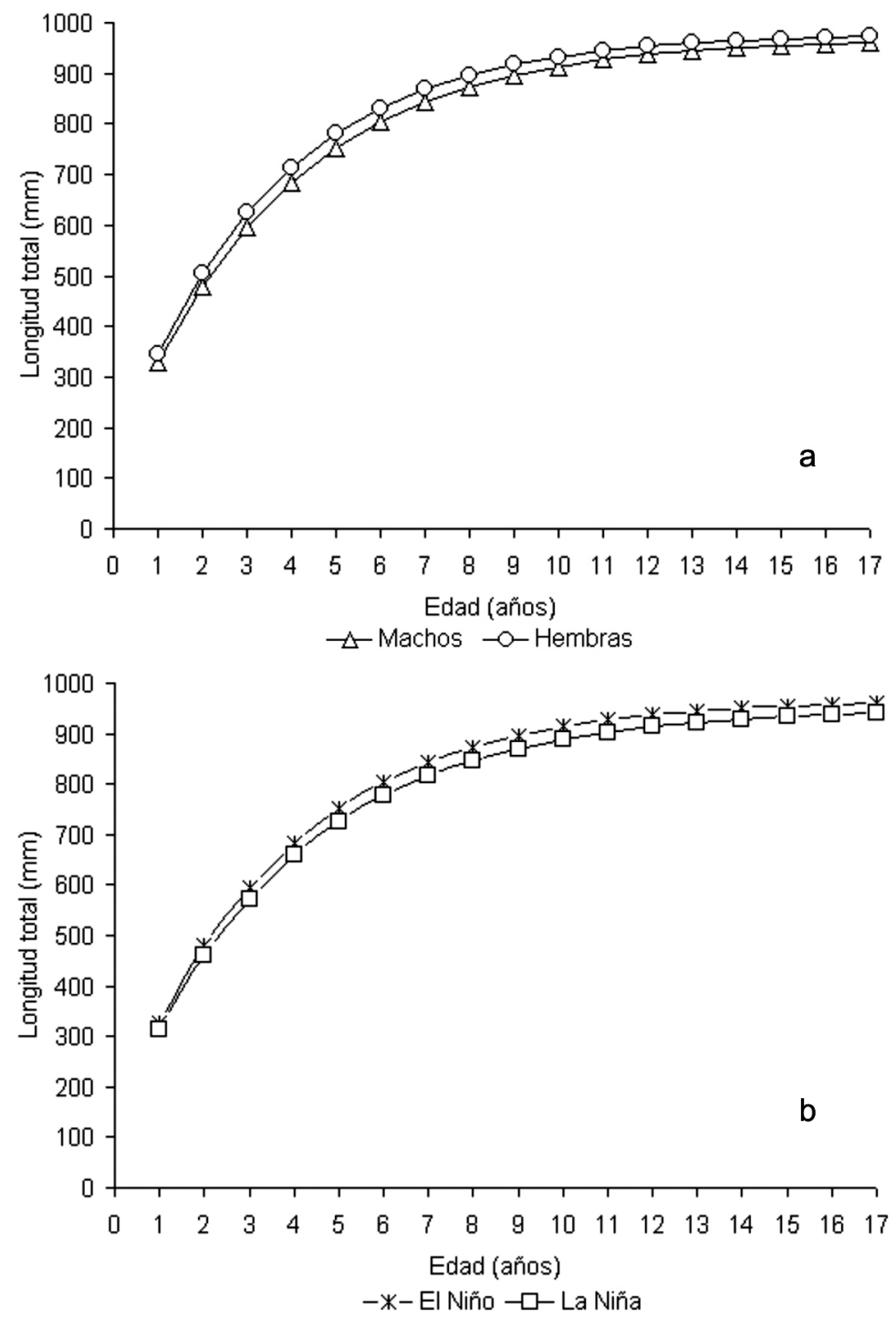

Figura 4. Curvas de crecimiento de $L$. argentiventris ajustadas a las estimaciones de los parámetros de crecimiento de la ecuación de Von Bertalanffy. a) Machos y hembras, b) Fenómeno de El Niño y La Niña.

Figure 4. L. argentiventris growth curves fitted to the growth parameters estimated of the Von Bertalanffy equation. a) Males and females, b) El Niño and La Niña. 


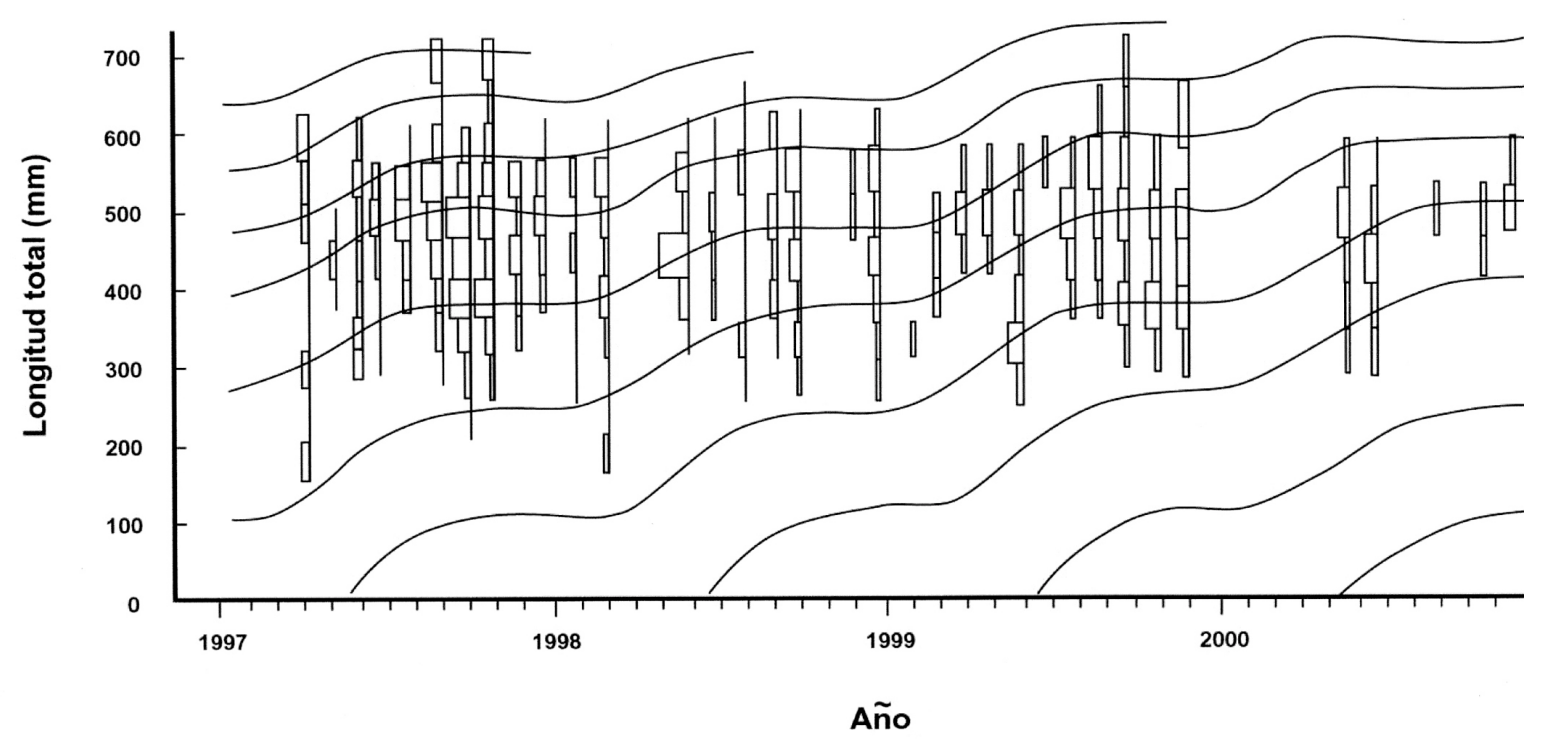

Figura 5. Curvas de crecimiento ajustadas a las distribuciones mensuales de frecuencias de tallas de $L$. argentiventris en el período de estudio.

Figure 5. L. argentiventris growth curves fitted to the monthly length frequency distribution in the study period.

La CPUE por año descendió a más de la mitad de 1997 a 1998, mantuvo valores similares en 1998 y 1999, y descendió drásticamente en el 2000 (Tabla 2). El comportamiento mensual por año de la CPUE mostró máximos de abundancia especialmente en mayo de 1998 y 1999, y en septiembre de 1997, 1998 y 1999. En 1997 los valores de la CPUE sobresalieron con respecto a los otros años entre julio y octubre, siendo estos los valores más altos de todo el período de estudio. El año 2000 mostró valores bajos en general, con un leve máximo en mayo. A partir de 1998 se observan meses en que $L$. argentiventris no fue capturado durante el período de muestreo (CPUE $=0 \mathrm{~g} /$ anzuelo), haciéndose esto más evidente en el 2000, donde las capturas fueron escasas (Fig. 6).

\section{DISCUSIÓN}

Este estudio no mostró una clara distribución batimétrica, pero se pudo observar una tendencia de individuos pequeños a escasa profundidad e individuos grandes a mayor profundidad. En Bahía Solano, Colombia (Torres, 1996) se capturaron individuos de $L$. argentiventris a profundidad prome- dio de 25,2 m, con tallas menores en comparación con los capturados en Gorgona a partir de los 40,2 $\mathrm{m}$ de profundidad. También se ha observado en jornadas de buceo autónomo en Gorgona, juveniles de L. argentiventris y $L$. guttatus a escasa profundidad $4,95 \mathrm{~m}$ en promedio ( 15 pies), normalmente asociados a sustratos rocosos que pueden ofrecer refugio (A. Suárez, com. pers.). Además, se han reportado juveniles de L. argentiventris en arrecifes coralinos, pozas litorales, estuarios, bahías y desembocaduras de ríos formando cardúmenes (Rubio, 1988; Fisher et al., 1995; obs. pers.), mientras que los juveniles de L. guttatus (Suárez, 1992) y L. jocu (GarcíaCagide et al., 1999) se han reportado en raíces de manglares y esteros, moviéndose hacia aguas más profundas conforme van creciendo. Este comportamiento ocurre en otras especies costeras del océano Pacífico colombiano, donde sus primeras etapas de vida se encuentran en el manglar, esteros o zonas rocosas de la costa, protegiéndolos de grandes predadores y ofreciendo alimento, para moverse después mar afuera una vez que crecen. Las diferencias en la talla promedio de captura en diferentes lugares y a diferentes profundidades es un argumento más que apoya la eventual distribución batimétrica por tallas de esta especie. En Gorgona, 
la talla promedio de captura (482 mm LT) no varió en forma notoria a través de los años (Tabla 2), y fue mayor que el rango de tallas más frecuente en Bahía Solano (270-360 mm LT; Torres, 1996), y que la reportada en México (Cruz-Romero et al., 1996), de 200-280 mm LS (258-348 mm LT con base en la ecuación (7)), donde se analizaron capturas de varios artes de pesca (mallas, trampas, espinel) que pudieron obtener un amplio rango de tallas, y aun así estuvo por debajo de la encontrada en Gorgona. Sin embargo, Rubio (1987) reporta la mayor talla promedio de captura para el Pacífico colombiano en general $(550 \mathrm{~mm})$.

La talla máxima observada en este estudio (700 mm LT) supera la talla máxima dada por Franke \& Acero (1992) para la isla Gorgona (660 mm) que es la misma reportada por Fischer et al. (1995) para la especie. No obstante lo anterior, Rubio (1988) indica una talla máxima de $800 \mathrm{~mm}$ que a juicio de otros autores es algo exagerada.

L. argentiventris no mostró períodos reproductivos claros durante este estudio; mayo, junio, julio, agosto, noviembre y diciembre tuvieron máximos reproductivos, unos más intensos que otros, y coincidiendo muy poco de año a año (Fig.
2). Este comportamiento se puede deber a los cambios medioambientales generados por los fenómenos de El Niño y La Niña; en 1997 hubo tres máximos reproductivos; en 1998 dos; mientras que en 1999 y 2000 solo uno. Se observa actividad reproductiva más intensa durante el fenómeno de El Niño que durante el fenómeno de La Niña; sin embargo, con la actual información no se alcanzan a comprobar dichas diferencias y es necesario hacer estudios más detallados al respecto. Se ha demostrado por lo pronto, que tanto El Niño como La Niña afectaron de manera similar el comportamiento reproductivo de la carduma (Cetengraulis mysticetus) en el Pacífico colombiano, observando varios máximos reproductivos en el año, siendo que en condiciones normales tiene uno solo y es considerada como desovante total (Beltrán-León, 2002).

Por otro lado, Torres (1996) estima que $L$. argentiventris presenta tres pulsos de desove en Bahía Solano, los dos primeros menos fuertes entre mayo y agosto, y uno que asume como el máximo o de desove masivo entre noviembre y marzo, indicando que la especie presenta un desove prolongado de aproximadamente 10 meses, muy similar a como se presenta en otras especies del género

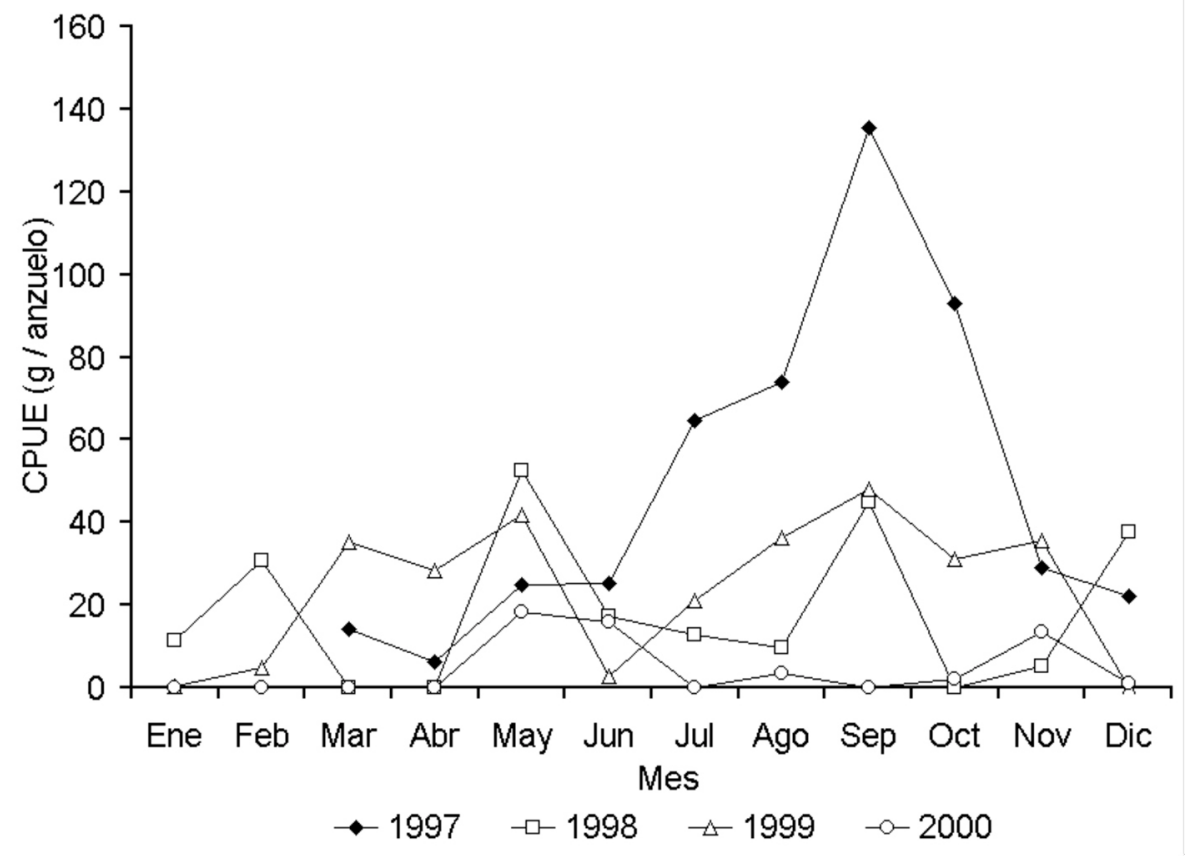

Figura 6. Captura por unidad de esfuerzo (CPUE) por año para L. argentiventris en el Parque Nacional Natural Gorgona.

Figure 6. Capture per unit effort (CPUE) by year for L. argentiventris in the National Natural Park Gorgona. 
Lutjanus (Sent et al., 1973; Manickchand-Heileman \& Phillip, 1996; García-Cagide et al., 1999). Sin embargo, Cruz-Romero et al. (1996), identificaron dos períodos reproductivos claros en México uno en abril-mayo y otro en septiembre-noviembre, observando diferencias en el patrón reproductivo de las poblaciones de Colombia (desove prolongado) y México (desove demarcado). Aunque Grimes (1986) reporta diferencias en el patrón de reproducción entre las poblaciones continentales e insulares de lutjanidos, donde las poblaciones insulares presentan pulsos de desove en primavera y otoño, mientras que las poblaciones continentales presentan un período prolongado de reproducción, esto no coincide para el caso de L. argentiventris. Dado su amplio rango de distribución en el Pacífico oriental, es posible que la especie adopte diferentes estrategias reproductivas, de acuerdo con la zona en que se encuentre. Así, la estrategia de un período reproductivo prolongado en Colombia le permite mayor éxito en la reproducción de acuerdo con las condiciones medioambientales del Pacífico colombiano, mientras que en México las temporadas demarcadas de reproducción van más acorde con las condiciones medioambientales de esas latitudes.

La talla promedio de madurez sexual $(515 \mathrm{~mm}$ LT) difiere de lo estimado por Cruz-Romero et al. (1996) (190-200 mm LS; 217-228 mm LT con base en la ecuación (7)). En el presente estudio no se registró gran cantidad de individuos de tallas menores, determinándose que el ejemplar más pequeño en estado avanzado de desarrollo gonadal midió 310 mm LT, por encima de la talla media encontrada en México. Se sugiere hacer estudios más intensivos sobre la reproducción de la especie en la zona, incluyendo el análisis histológico de las gónadas.

La proporción de sexos de $L$. argentiventris estimada en este estudio $(1: 1,2)$ difiere de lo estimado por Torres (1996) $(2: 1,5)$ y Cruz-Romero et al. (1996) (1:0,9); en estos estudios se observa ligera mayor presencia de machos que de hembras, mientras que en Gorgona tiende a ocurrir lo contrario. Las causas de estas diferencias no son claras aún y es necesario hacer estudios más detallados a este respecto, donde se analice la distribución de sexos en términos de la talla y la profundidad.

Considerando los datos obtenidos, $L$. argentiventris sigue la tendencia de varias especies del género Lutjanus donde no existen diferencias significativas en la relación longitud-peso entre hembras y machos (Sent et al., 1973; Kaunda-Arara \&
Ntiba, 1998; Carrillo de Albornoz, 2000; Burton, 2001), exceptuando algunas especies del mismo género en la gran barrera de coral australiana, en las cuales sí se observaron tales diferencias (McPherson \& Squire, 1992; Newman et al., 1996, 2000; Newman, 2002).

Las estimaciones de los parámetros de crecimiento de machos y hembras no mostraron grandes diferencias entre sí, aunque sí una ligera tendencia de las hembras a crecer más $\left(\mathrm{L}_{\infty}\right)$ y más rápido $(\mathrm{K})$ que los machos (Fig. 4 a). Mayores valores de $\mathrm{K}$ de hembras sobre machos se han observado igualmente en otras especies del género Lutjanus (McPherson \& Squire, 1992; Rocha-Olivares, 1997; Newman et al., 2000; Newman, 2002) aunque en estos casos los valores de $\mathrm{L}$ han sido menores en las hembras. Es necesario obtener en el futuro rangos de tallas más amplios para dilucidar posibles diferencias en el crecimiento de hembras y machos de esta especie.

Tampoco se observaron grandes diferencias al analizar los parámetros de crecimiento estimados en los períodos de El Niño y La Niña, donde el evento cálido observó valores ligeramente más altos en los parámetros de crecimiento que los del evento frío (Fig. 4b). Esto sugiere que de haber cambios en el crecimiento por uno u otro fenómeno, dichos cambios se manifiestan de forma parecida en esta especie. Estimaciones en condiciones medioambientales normales en la misma zona permitirán hacer comparaciones con las actuales estimaciones y dar una idea de la influencia de estos fenómenos en el crecimiento de la especie.

La relación longitud-peso y los parámetros de crecimiento estimados en este estudio fueron también diferentes a lo estimado por Torres (1996) (Tabla 3), lo cual puede estar asociado a una diferencia en las distribuciones de frecuencias de tallas de las muestras tomadas en cada lugar. Sin embargo, en este estudio se tuvo una mejor representación de especímenes con tallas mayores, lo que hace tener una mejor estimación de la longitud asintótica, haciendo a su vez la estimación de $\mathrm{K}$ menos dependiente de $\mathrm{L}_{\infty}$.

Se observaron también diferencias en las estimaciones de la relación longitud-peso y en los parámetros de crecimiento entre Gorgona (Colombia) y México (Cruz-Romero et al., 1996) (Tabla 3). Así, los valores de $\mathrm{L}_{\infty} \mathrm{y} \mathrm{W}_{\infty}$ resultaron mayores en L. argentiventris de Gorgona; K denota un crecimiento más rápido en dicho lugar comparado con 
lo reportado para México, y con los de otras especies del mismo género en zonas tropicales y semitropicales que alcanzan tallas similares (Liu \& Yeh, 1991; McPherson \& Squire, 1992; Manickhand-Heileman \& Phillip, 1996; RochaOlivares, 1998; Carrillo de Albornoz, 1999; Burton, 2001), cuyas estimaciones no sobrepasaron de 0,17 años $^{-1}$. Es posible que las estimaciones estén influidas por oscilaciones en el crecimiento (Fig. 5), como respuesta a los cambios ocasionados por los fenómenos de El Niño y La Niña, ya que no hubo un patrón claro de máximos reproductivos que pudieran ocasionar estas oscilaciones.

Para calcular M en especies del género Lutjanus se han utilizado las fórmulas de Pauly (1980), Hoenig (1983) y Ralston (1987) en varios estudios (Acosta \& Appeldoorn, 1992; Newman et al., 1996, 2000; Rocha-Olivares, 1998; Burton, 2001; Newman, 2002). En general, se ha observado diferencia en la estimación de acuerdo con el método utilizado. Así, Newman et al. (1996) anotan que el $82,6 \%$ de los estudios de lutjanidos han calculado M de acuerdo con la fórmula de Pauly (1980), aun- que repara en que las estimaciones basadas en fórmulas como las de Pauly (1980) y Ralston (1987) deben ser aplicadas con precaución en especies de larga vida, ya que tienden a sobrestimar M. Por otra parte, Newman et al. (2000) reportan cómo la ecuación de Hoenig (1983) provee estimaciones más confiables de $\mathrm{M}$ en lutjanidos si se dispone de muestras que abarquen un amplio rango de tallas (edades); argumentan además, que la ecuación de Hoenig proviene de gran cantidad de datos de especies no explotadas e incluye varias especies con rango amplio de longevidades, mientras que la ecuación de Pauly incorpora datos de relativamente pocas especies de larga vida. Dado que no se tenían estimaciones de edad para $L$. argentiventris en Gorgona, M se estimó con las fórmulas de Ralston (1987) y Pauly (1980), los cuales fueron parecidos al estimativo de $2^{*} \mathrm{~K}(0,4$; donde $\mathrm{K}$ es la constante de crecimiento de la ECVB) que según Ralston (1987), es un buen estimativo preliminar de M para lutjanidos en ausencia de otra información. Sin embargo, es posible que $L$. argentiventris, tal como L. peru (RochaOlivares, 1998) tenga diferencias en la tasa de mor-

Tabla 3. Comparación de los parámetros de crecimiento $\left(L_{\infty}, W_{\infty}, K, t\right)$, relación longitud-peso (a, b), y mortalidad natural $(\mathrm{M})$ en tres estudios realizados sobre $L$. argentiventris.

Table 3. Comparison of growth parameters $\left(L_{\infty}, W_{\infty}, K, t\right)$, length-weight relationship (a, b), and natural mortality $(\mathrm{M})$ in three studies about $L$. argentiventris.

\begin{tabular}{|c|c|c|c|}
\hline & $\begin{array}{c}\text { Cruz-Romero } \\
\text { et al. (1996) }\end{array}$ & Torres (1996) & Este estudio \\
\hline \multirow[t]{2}{*}{$L_{\infty}$} & $\begin{array}{l}685 \text { mm (LS) } \\
\text { ELEFAN 1 } \\
\text { Pauly (1987) }\end{array}$ & & \\
\hline & $802 \mathrm{~mm}(\mathrm{LT}) *$ & $\begin{array}{c}720 \mathrm{~mm} \\
\text { ELEFAN } 1 \\
\text { Gayanilo } \text { et al. (1996) }\end{array}$ & $\begin{array}{c}950 \text { mm (LT) } \\
\text { ELEFAN 1 } \\
\text { Gayanilo et al. (1996) }\end{array}$ \\
\hline$W_{\infty}$ & $6676 \mathrm{~g}$ & $5514,5 \mathrm{~g}$ & $12218 \mathrm{~g}$ \\
\hline$K$ & $\begin{array}{c}0,155 \text { año }^{-1} \\
\text { ELEFAN } 1 \\
\text { Pauly (1987) }\end{array}$ & $\begin{array}{c}0,6 \text { año }^{-1} \\
\text { ELEFAN } 1 \\
\text { Gayanilo et al. }(1996)\end{array}$ & $\begin{array}{c}\text { 0,20 año } \\
\text { ELEFAN } 1 \\
\text { Gayanilo } \text { et al. }(1996)\end{array}$ \\
\hline$t_{o}$ & - & $\begin{array}{c}-0,212 \\
\text { Pauly (1983) }\end{array}$ & $\begin{array}{c}-0,722 \\
\text { Pauly (1983) }\end{array}$ \\
\hline a & 0,070 & 0,01576 & $6,259 * 10^{-6}$ \\
\hline $\mathrm{b}$ & 2,75 & 2,98 & 3,12 \\
\hline
\end{tabular}

* Con base en la ecuación (7) del presente estudio 
talidad de acuerdo al sexo, dada su diferencia en la proporción de sexos $(1: 1,2)$ y la tendencia de las hembras a ser más comunes a tallas mayores.

L. argentiventris fue una especie representativa en las capturas de las faenas experimentales realizadas durante todo el período de estudio, junto con Epinephelus acanthistius (Serranidae) y Brotula clarkae (Ophidiidae), especies de mayor frecuencia en la zona y similar importancia económica en el Pacífico colombiano. El análisis de la CPUE, como índice de abundancia, muestra cómo este disminuye considerablemente de año a año en un determinado mes (Fig. 6). Las variaciones de la CPUE se deberían a los siguientes factores: (i) Migraciones: En la zona sur del Pacífico colombiano la época de pesca de pargo (Lutjanus spp, y Holopagrus sp.) se ha ubicado tradicionalmente entre mayo y julio, la cual se reconoce como la de mayores capturas de acuerdo a los pescadores artesanales, lo que hace suponer que los lutjanidos tienen un patrón de migración desde y hacia la zona. Observaciones en otras especies del género Lutjanus sugieren que algunas forman agregaciones para el desove ( $L$. synagris, L. jocu y L. griseus) mientras que otras tienen épocas de desove restringidas sin aparentes migraciones por reproducción (Wicklund, 1969; Carter \& Perrine, 1994; Domeier et al., 1996). (ii) Efecto de los fenómenos de El Niño y La Niña: durante El Niño se observaron las mayores capturas de L. argentiventris en la zona, las cuales se redujeron en los siguientes años, pero manteniéndose estables durante el período de La Niña, para reducirse drásticamente en el 2000. Es posible que la considerable disminución observada en el 2000 haya sido un efecto acumulado de El Niño y La Niña que esta vez se dieron uno seguido del otro, o bien un efecto del fenómeno frío solamente, causando migraciones fuera del patrón normal, afectando el tamaño y/ o comportamiento del stock desovante, de juveniles y/o reclutas. El impacto de El Niño y La Niña sobre especies de peces demersales del Pacífico colombiano no ha sido reportado hasta ahora. Zapata (2002) reporta los efectos de estos fenómenos en ciertas pesquerías del océano Pacífico colombiano, citando un aumento de las capturas de camarones durante El Niño, los cambios en la distribución y comportamiento de especies pelágicas como Cetengraulis mysticetus, Opisthonema sp. y Coryphaena hippurus. Beltrán-León (2002) hace análisis más detallados de los efectos de estos fenómenos en la distribución y abundancia de $C$. myticetus para la misma zona. En Perú se ha visto cómo las especies demersales tienden a migrar hacia el sur (Espino, 1991); en Ecuador y Perú el camarón también ha sido más abundante de lo normal durante el fenómeno de El Niño (Jiménez \& Herdson, 1984; Espino, 1991).

Aunque las estimaciones aquí reportadas pudieron verse influidas por la selectividad del arte de pesca y los fenómenos de El Niño y La Niña, se puede evidenciar una clara diferencia entre las poblaciones de L. argentiventris de la isla Gorgona en Colombia y las de Manzanillo y Boca de Apiza en México; esto se puede deber a diferencias ecológicas tales como la abundancia de la especie, la diversidad y/o dominancia de sus predadores y/o presas naturales, las posibles diferencias genéticas entre las poblaciones, las diferencias en la intensidad pesquera de cada lugar y diferencias en la respuesta de cada una de las poblaciones a los cambios ambientales generados por El Niño y/o La Niña, los cuales de hecho tuvieron manifestaciones diferentes en cada una de estas regiones geográficas.

Los parámetros de crecimiento de $L$. argentiventris han sido estimados hasta ahora sobre la base del análisis de las distribuciones de frecuencias de tallas. Para obtener estimaciones más precisas es necesario asegurar un amplio rango de tallas representadas en la muestra e intentar otros métodos tales como los basados en el análisis de estructuras duras (otolitos especialmente). Se ha demostrado una alta confiabilidad y precisión de estos métodos para peces del genero Lutjanus de regiones tropicales y semitropicales (ManickhandHeileman, 1996; Newman et al., 1996, 2000; Rocha-Olivares, 1998; Burton, 2001; Newman, 2002), o bien la combinación del análisis de frecuencia de tallas y el análisis de otolitos (Morales-Nin, 1989, 1990).

Aunque la población de L. argentiventris de Gorgona no está sujeta a una explotación comercial por ser esta zona un Parque Nacional Natural, la información aquí recopilada puede tener importantes implicaciones de manejo pesquero, pues esta especie es de crecimiento moderadamente lento, de aparentemente larga vida y una tasa de mortalidad natural baja, que la hacen fácilmente vulnerable a una sobrepesca. Trabajos más detallados se requieren para complementar el conocimiento aportado sobre $L$. argentiventris en los diferentes aspectos tratados en el presente estudio. 


\section{AGRADECIMIENTOS}

Este estudio se realizó gracias al apoyo logístico y financiero de la Unidad Administrativa Especial del Sistema de Parques Nacionales Naturales de Colombia (UAESPNN), enmarcado dentro del Proyecto Parques del Chocó Biogeográfico. Especiales agradecimientos a los biólogos Claudia Acevedo y Gustavo Mayor, Directora y profesional universitario del PNN Gorgona respectivamente, al personal del parque en general, y a los señores Gabino Ibarbo y Miguel Castro, con quienes se realizaron las faenas experimentales de pesca.

\section{REFERENCIAS}

Acosta, A. \& R.S. Appeldoorn. 1992. Estimation of growth, mortality and yield per recruit for Lutjanus synagris (Linnaeus) in Puerto Rico. Bull. Mar. Sci., 50(2): 282-291.

Allen, G.R. 1985. Snappers of the world. An annotated and illustrated catalogue of lutjanid species known to date. FAO Fisheries Synopsis, 125(6): 1-208.

Alvarez, R. 1980. A specimen of Lutjanus argentiventris (Peters) lacking pelvic fins. J. Fish. Biol., 16(5): 563-564.

Alvarez, R. 1984. Teratological case in one lutjanid (Pisces: Lutjanidae) from southern Sinaloa, México. An. Inst. Biol. Univ. Nac. Autón. Méx. Zool., 54(1): 229-235.

Beltrán-León, B.S. 2002. Changes in the distribution, abundance and spawning season of the anchovy Cetengraulis mysticetus, in the Pacific Ocean off Colombia during the Events of El Niño and La Niña. Invest. Mar., Valparaíso, 30(1), 106-107.

Burton, M.L. 2001. Age, growth, and mortality of gray snapper, Lutjanus griseus, from the east coast of Florida. US Fish. Bull., 99(2): 254-265.

Carrillo de Albornoz, C. 2000. Assessment and growth of the Lutjanus chrysurus at the southeastern shelf west of Cuba. Rev. Invest. Mar. México City, 21(1-3): 45-52.

Carter J. \& D. Perrine. 1994. A spawning aggregation of dog snapper Lutjanus jocu (Pisces: Lutjanidae) in Belize, Central America. Bull. Mar. Sci., 55(1): 228-234.
Cruz-Romero, M., E.A. Chávez, E. Espino \& A. García. 1996. Assessment of a snapper complex (Lutjanus spp.) of the eastern tropical Pacific. En: F. Arreguin-Sanchez, J.L. Munro, M.C. Balgos \& D. Pauly (eds.). Biology, fisheries and culture of tropical groupers and snappers. ICLARM Conf. Proc. No. 48, Manila, pp. 324-330.

Domeier, M.L., C. Koenig \& F. Coleman. 1996. Reproductive biology of the gray snapper (Lutjanus griseus), with notes on spawning for other western Atlantic snappers (Lutjanidae). En: F. ArreguinSanchez, J.L. Munro, M.C. Balgos \& D. Pauly (eds.). Biology, fisheries and culture of tropical groupers and snappers. ICLARM Conf. Proc. No. 48, Manila, pp. 189-201.

Espino, M. 1991. El Niño: su impacto sobre los peces demersales de Perú. Perú. Pesq., 3(6): 19-24.

Fischer, W., F. Krupp, W. Schneicler, C. Sornoner, K.E. Carpenter \& V.H. Nsem. 1995. Guía FAO para la identificación de especies para los fines de la pesca; Pacífico Centro - Oriental. Vol. II y III. Peces FAO. Roma, 1687 pp.

Franke, R. y P.A. Acero. 1992. Lutjanid fishes of the Gorgona Park, Colombian Pacific (Osteichthyes: Lutjanidae). Rev. Biol. Mar., 27(1): 59-71.

García-Cagide, A.R., R. Claro \& J.P. GarcíaArteaga. 1999. Biology of the dog snapper Lutjanus jocu in the NE and SW zones of Cuban shelf. I. Distribution, habitat, reproduction and dynamics of the morphophysiological indicators. Rev. Invest. Mar. Mexico City, 20(1-3): 22-29.

Gayanilo, Jr. F.C., P. Sparre \& D. Pauly. 1996. FAOICLARM stock assessment tools. International Center for Living and Aquatic Resources Management.-Food and Agriculture Organization of the United Nations. FAO Computerized Information Series Fisheries, Rome.

Hoenig, J.M. 1983. Empirical use of longevity data to estimate mortality rates. US Fish. Bull., 82(1): 898-902.

Jiménez, R. \& D. Herdson. 1984. Efectos de "El Niño" 1982-1983 sobre los recursos pesqueros en Ecuador. Rev. Com. Perm. Pacífico Sur, 15: 269291.

Kaunda-Arara, B. \& M.J. Ntiba. 1998. Lengthweight relationship, condition and food of Lutjanus fulviflamma (Forsskal, 1785) (Pisces: Lutjanidae) 
from Kenya inshore marine waters. International conference on African fishes and fisheries diversity and utilisation, Grahamstown, South Africa, 1318 Sept. 1998. Paradi Association and the Fisheries Society of Africa; Grahamstown, South Africa FISA 1988, 288 pp.

King, M. 1995. Fisheries biology, assessment and management. Fishing News Books. Blackwell Science, London, 341 pp.

Liu, C.C. \& S.Y. Yeh. 1991. Age determination and growth of red emperor snapper (Lutjanus sebae) in the Arafura Sea off north Australia. Acta Oceanogr. Taiwan, 26: 36-52.

McPherson, G.R. \& L. Squire. 1992. Age and growth of three dominant Lutjanus species of the Great Barrier Reef inter-reef fishery. Asian-Fish. Sci., 5: 25-36.

Manickchand-Heileman, S.C. \& D.A.T. Phillip. 1996. Reproduction, age and growth of the Caribbean red snapper (Lutjanus purpureus) in waters off Trinidad and Tobago. En: F. ArreguinSanchez, J.L. Munro, M.C. Balgos \& D. Pauly (eds.). Biology, fisheries and culture of tropical groupers and snappers. ICLARM Conf. Proc. No. 48, Manila, pp. 137-149.

Morales-Nin, B. 1989. Growth determination of tropical marine fishes by means of otolith interpretation and length frequency analysis. Aquat. Living Resour., 2: 241-253.

Morales-Nin, B. \& S. Ralston. 1990. Age and growth of Lutjanus kasmira (Forskal) in Hawaiian waters. J. Fish. Biol., 36(2): 191-203.

Newman, S.J. 2002. Growth rate, age determination, natural mortality and production of potential of the scarlet seaperch, Lutjanus malabaricus Schneider 1801 , off the Pilbara coast of north-western Australia. Fish. Res., 58(2002): 215-225.

Newman, S.J., D.McB. Williams \& G.R. Russ. 1996. Age validation, growth and mortality rates of the tropical snappers (Pisces: Lutjanidae) Lutjanus adetii (Castelnau, 1873) and L. quinquelineatus (Bloch, 1790) from the central Great Barrier Reef, Australia. Mar. Freshwat. Res., 47(4): 575-584.

Newman, S.J., M. Cappo \& D.McB. Williams. 2000. Age, growth and mortality of the stripey, Lutjanus carponotatus (Richardson) and the brown-stripe snapper, L. vitta (Quoy and Gaimard) from the cen- tral Great Barrier Reef, Australia. Fish. Res., 48(3): 263-275.

Pauly, D. 1980. On the interrelationships between natural mortality, growth parameters, and mean environmental temperature in 175 fish stocks. J. Cons. int. Explor. Mer, 39(2): 175-192.

Pauly, D. 1983. Algunos métodos simples para la evaluación de recursos pesqueros tropicales. FAO Doc. Téc. Pesca, 234: 49 pp.

Ralston, S. 1987. Mortality rates of snappers and groupers. En: J.J. Polovina \& S. Ralston (eds.). Tropical snappers and groupers: biology and fisheries management. Westview Press. Boulder, CO, pp. 375-404.

Rocha-Olivares, A. 1998. Age, growth, mortality, and population characteristics of the Pacific red snapper, Lutjanus peru, off the southeast coast of Baja California, Mexico. US Fish. Bull., 96(3): 562-574.

Rubio, E.A. 1987. Lista sistemática de los peces costeros y de profundidad del Pacífico colombiano. Universidad del Valle, Cali, Colombia. 192 pp.

Rubio, E.A. 1988. Peces de importancia comercial del Pacífico colombiano. Cent. Inv. Mar. Est. Univ. del Valle, Contribución 1, Cali, Colombia, 499 pp.

Sent, T., P. Kungvankij \& S.M. Tan. 1973. Biological study of red snapper, Lutjanus sanguineus. Southeast Asian Fish. Dev. Cent., Changi Point (Singapore) Mar. Fish. Res. Dep. Tech. Sem. On South China Sea Fisheries Resources, Bangkok (Thailand), 21 May 1973, 19 pp.

Serrano-Pinto, V. \& J. Caraveo-Patiño. 1999. Survival of amarillo snapper Lutjanus argentiventris (Peters 1869) at different salinities in captivity. Aquacult. Res., 30(6): 467-470.

Suárez, A. 1992. Estudio preliminar sobre la biología (alimentación, reproducción y crecimiento) de Lutjanus guttatus en el Pacífico Colombiano. Tesis de Grado. Universidad del Valle, Facultad de Ciencias, Departamento de Biología. Cent. Documentación, Cali, Colombia, 100 pp.

Torres C.A. 1996. Aspectos biológico-pesqueros del pargo planero Lutjanus argentiventris (Peters 1869) y reconocimiento sobre la pesca artesanal en el municipio de Bahía Solano (Chocó-Colombia). Informe Técnico Instituto Nacional de Pesa y Acuicultura INPA, Colombia, 16 pp. 
Wicklund, R. 1969. Observations on spawning of lane snapper. Underwater Nat., 6(2): 40.

Recibido: 28 febrero 2003; Aceptado: 20 julio 2004.
Zapata, L.A. 2002. Effects of El Niño and La Niña on the fisheries of the Colombian Pacific. Invest. Mar., Valparaíso, 30(1): 205-206. 